\title{
CDK1 and PLK1 coordinate the disassembly and reassembly of the nuclear envelope in vertebrate mitosis
}

\author{
Ines J de Castro ${ }^{1,2, *}$, Raquel Sales Gil1,* ${ }^{1,}$ Lorena Ligammari ${ }^{1}$, Maria Laura Di Giacinto ${ }^{1}$ \\ and Paola Vagnarelli ${ }^{1}$ \\ ${ }^{1}$ College of Health and Life Science, Research Institute for Environment Health and Society, Brunel University London, UB8 \\ $3 \mathrm{PH}, \mathrm{UK}$ \\ ${ }^{2}$ Current address: Department of Infectious Diseases, Integrative Virology, University Hospital Heidelberg and German \\ Center for Infection Research (DZIF), 69120 Heidelberg, Germany \\ *These authors contributed equally to this work
}

Correspondence to: Paola Vagnarelli, email: Paola.Vagnarelli@Brunel.ac.uk

Keywords: nuclear envelope; polo Like Kinase (PLK1); nuclear pore complex (NPC); lamin A; micronuclei; chromosome

Received: August 30, $2017 \quad$ Accepted: October 31, $2017 \quad$ Published: December 23, 2017

Copyright: Castro et al. This is an open-access article distributed under the terms of the Creative Commons Attribution License 3.0 (CC BY 3.0), which permits unrestricted use, distribution, and reproduction in any medium, provided the original author and source are credited.

\section{ABSTRACT}

Micronuclei (MN) arise from chromosomes or fragments that fail to be incorporated into the primary nucleus after cell division. These structures are a major source of genetic instability caused by DNA repair and replication defects coupled to aberrant Nuclear Envelope (NE). These problems ultimately lead to a spectrum of chromosome rearrangements called chromothripsis, a phenomenon that is a hallmark of several cancers. Despite its importance, the molecular mechanism at the origin of this instability is still not understood. Here we show that lagging chromatin, although it can efficiently assemble Lamin A/C, always fails to recruit Nuclear Pore Complexes (NPCs) proteins and that Polo-Like Kinase (PLK1) negatively regulates NPC assembly. We also provide evidence for the requirement of PLK1 activity for the disassembly of NPCs, but not Lamina A/C, at mitotic entry. Altogether this study reveals the existence of independent regulatory pathways for Lamin A/C and NPC reorganization during mitosis where Lamin A/C targeting to the chromatin is controlled by CDK1 activity (a clock-based model) while the NPC loading is also spatially monitored by PLK1.

\section{INTRODUCTION}

During open mitosis the nuclear envelope breaks down at the end of prophase to allow the separation of sister chromatids and starts to re-assemble during anaphase (reviewed in [1]). Lamin A phosphorylation by cdc2/ cyclinB $[2,3]$ allows lamina disassembly at the beginning of mitosis while multiple kinases appear to contribute to the disassembly of the NPC via phosphorylation of a crucial target, Nup98 [4]. During mitotic exit, NE reformation (NER) occurs as a sequential and multi-step process where NUPs assemble to the chromatin earlier than Lamin A [5] but the overall mechanism is still poorly understood (recently reviewed in [6] and [7]).

A few studies have shown that this mechanism is regulated by the concerted action of protein phosphatase 1 (PP1) $[8,9]$ and 2A (PP2A) [10]. This ordered assembly during NER suggests that sequential dephosphorylation steps must occur to allow macromolecular complexes to form and target them to the correct place at the right time. This could be achieved by activation of different phosphatases at different times during mitotic exit (clock model), by the position of the segregating chromatin within the anaphase cell (spatial model) or a combination of both. However, the molecular effectors are still not known. Recent studies in Drosophila have shown that an Aurora B gradient appears to regulate a surveillance mechanism that prevents both chromosome decondensation and NER until effective separation of sister chromatids is achieved [11] and to delay Lamin A re-assembly [12].

Here we show for the first time that, in vertebrates, Polo-like kinase 1 (PLK1) is also participating in the NER control pathway, and that it is essential both for timely disassembly of the NPC at the onset of mitosis and for preventing the loading of the NPC on the chromatin of lagging chromosomes during mitotic exit. 
This study proposes a sequential model for the NER where Lamin A targeting to the chromatin is under the control of decreasing cdc2/cyclinB activity (a clock-based model) while NPC loading is spatially regulated by PLK1 activity.

\section{RESULTS}

In order to understand the causes of MN instability, we have analysed the composition of the $\mathrm{NE}$ of $\mathrm{MN}$ in HeLa and U2OS cells by immunofluorescence.

While Lamin B1 was absent in all MN analysed in both cell lines (as previously reported [13]), we noticed heterogeneity among the $\mathrm{MN}$ in relation to the presence/ absence of Lamin A and Importin $\beta$ or the NPC marker $\mathrm{mAb} 414$, which recognizes several FG nucleoporins, with the majority of the MN lacking Importin $\beta$ and mAb414 (Supplementary Figure 1A, 1B). Although the absence of Lamin B1 could indicate that the $\mathrm{MN}$ have already been disrupted (as reported by Zhang et al. [14]), the presence or absence of Lamin A and other nuclear membrane (NM) components suggested that the loading of Lamin A could be uncoupled from that of nucleoporins. Indeed, Lamin A and NPC seem to have different loading times.

In order to understand how this variability could arise, we have focused on anaphase cells containing lagging chromosomes or chromatin bridges, the most common cause for MN formation [15]. NER starts in anaphase in a sequential manner and the order of events have been well documented [5]. For example, NUPs (as judged by mAb414 staining) and Importin $\beta$ recruitment precedes Lamin A and it starts loading at the poleward side of the segregating chromatids as shown in Supplementary Figure $1 \mathrm{C}-1 \mathrm{H}$.

Lagging chromatids arise from chromosome fragments without kinetochores or merotelic-attached chromosomes [16], dwelling in the middle of the anaphase spindle and causing a delay in division. Using several antibodies that recognize different NPC sub-complexes (mAb414 for FG containing NUPs, Nup93 for the inner ring complex, POM121 for the transmembrane NUPs and MEL28 for the Y complex [17]), we have revealed that chromatin present in the middle of the anaphase/ telophase spindle is competent for Lamin A assembly but fails to recruit either Importin $\beta$ or NPC components (Figure 1A-1F). Therefore, while a fraction of interphase $\mathrm{MN}$ are loaded with NPC components (Supplementary Figure 1B), lagging chromatin is not (Figure $1 \mathrm{~A}-1 \mathrm{~F}$ ) but still assembles Lamin A, thus indicating that the normal order of assembly (first NPC then Lamin A) is not maintained in this type of chromatin. These observations suggest that: 1) the process of Lamina re-assembly and NPC re-assembly after mitosis are independent of each other and they are under different regulatory systems; 2) recruitment of NPC components to the anaphase chromatin is negatively regulated directly or indirectly by factors (possibly kinases) acting at the central spindle or limited by the small amount of chromatin present. We discarded the latter hypothesis since we could identify cells with a substantial mass of lagging chromatin which was still negative for NPC components (Figure 2A).

We therefore started investigating a possible role for kinases as negative regulators of the NER. In Drosophila, it has recently been shown that Aurora B acts in anaphase to delay Lamin A and B re-assembly when chromatin is trapped in the midzone $[11,12]$. In contrast, our experiments indicate that Lamin A can successfully assemble on lagging chromatin (Figure 1A, 1B). We tested if inhibition of different kinases present in the anaphase midzone/midbody could allow NPC loading on lagging chromatin in vertebrates.

We inhibited PLK1 and Aurora B using wellestablished small molecules inhibitors for these kinases. BI2536 is a selective inhibitor for PLK1 [18, 19] and it has been extensively used to study the role of PLK1 during mitosis [20, 21-23]. In addition, the molecular details of BI2536 binding to PLK1 have been well characterized at atomic resolution [24]. Similarly, the Aurora B inhibitor ZM447439 [25] has been widely used to understand the role of this kinase during mitosis and its potency, selectivity, off-target profiles, and cellular efficacy have been assessed [26].

Inhibition of PLK1 or Aurora B was able to release the inhibitory effect and allowed part of the NPC loading onto the lagging chromatin (Figure 2B). While inhibition of Aurora B could have been expected based on the experiments conducted in Drosophila, the effect caused by PLK1 inhibition is surprising since this kinase has never been reported to act on NE reformation nor has it been shown to be able to generate a gradient in anaphase. Supporting our experiment, PLK1 was shown to be recruited to DNA tethers of acentric chromatin during cell division [27]. A further support to the implication of PLK1 in NPC regulation was provided by experiments where, by overexpression of mCherry:PLK1 in HeLa cells, we were able to see a significant reduction of NPC at the NE of $\mathrm{MN}$ (Figure 2C). This observation could either suggest that both kinases regulate a subset of NUPs or that one kinase activity depends on the other.

While it is known that Aurora B activates PLK1 at kinetochores in early mitosis both in Drosophila and human cells [28], its regulation in late mitosis is poorly understood. Only recently it has been suggested that in Drosophila, Map205 sequesters Polo kinase on central spindle microtubules and that, upon phosphorylation mediated by Aurora B, active Polo is recruited to the midbody where it targets substrates locally [29]. This model has the potential to serve as the regulatory mechanism for PLK1 and NPC loading. However, the vertebrate orthologue of Map205 is not known. We have therefore tested if a crosstalk between Aurora B and PLK1 could also exist in human cells. To this purpose we have 
treated HeLa cells for $30 \mathrm{~min}$ with the Aurora B inhibitor ZM447439 (ZM) and quantified the level of PhosphoPLK1 at the midbody relative to total PLK1. The results clearly show that Aurora B inhibition causes a significant decrease in Phospho-PLK1 at the midbody (Figure $2 \mathrm{D}, 2 \mathrm{E}$ ) thus indicating that, as in Drosophila, activation of PLK1 in the midbody depends on Aurora B. These results could potentially indicate that the phosphorylation status of PLK1 could affect NPC re-assembly. In fact, NPC deposition around the chromatin appears to occur away from the presence of PLK1ph, where PLK1ph levels are below a threshold (Figure 2F, 2G).

In order to further test the relevance of protein kinases in NE re-assembly, we analysed this process in
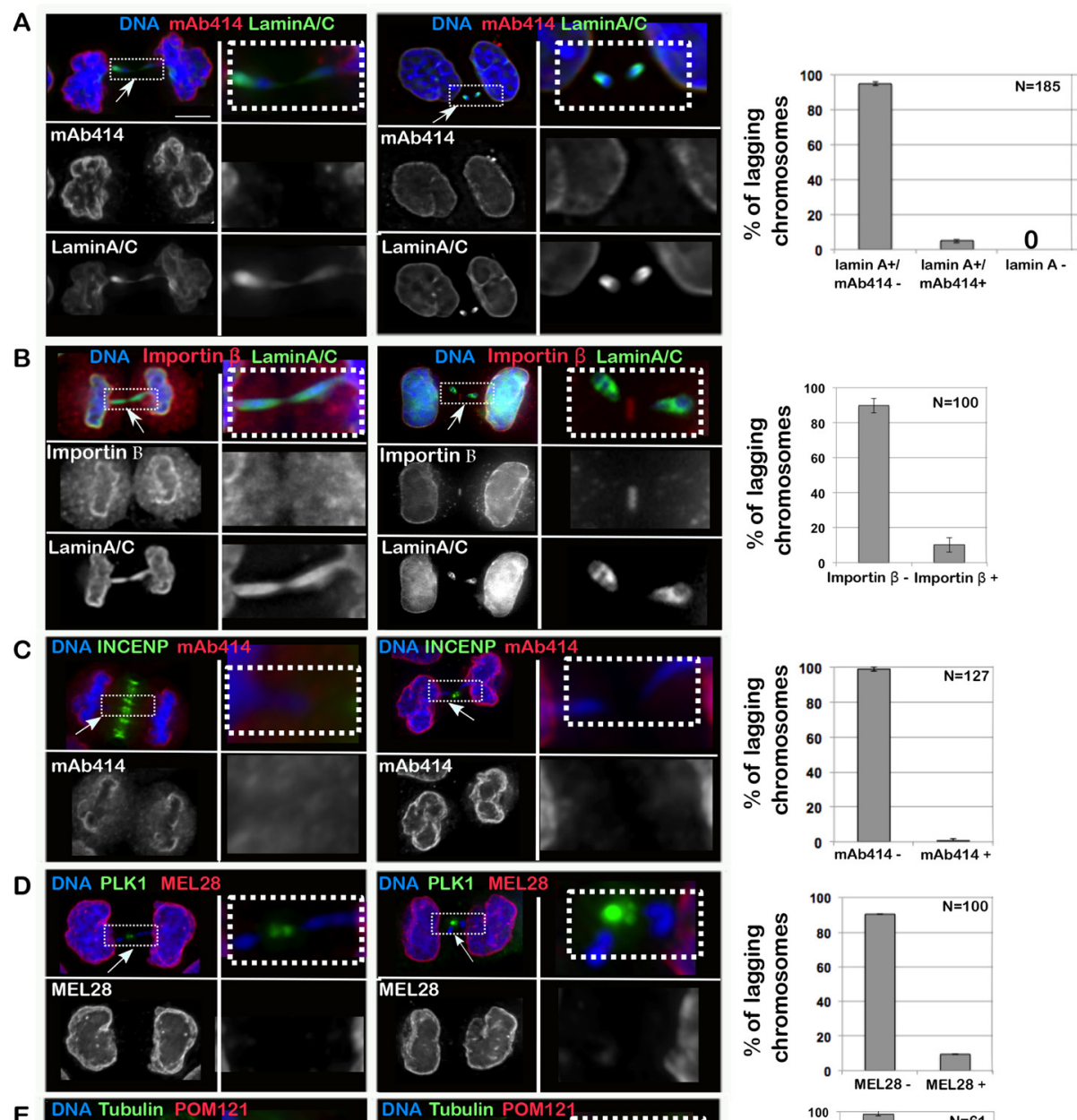

E
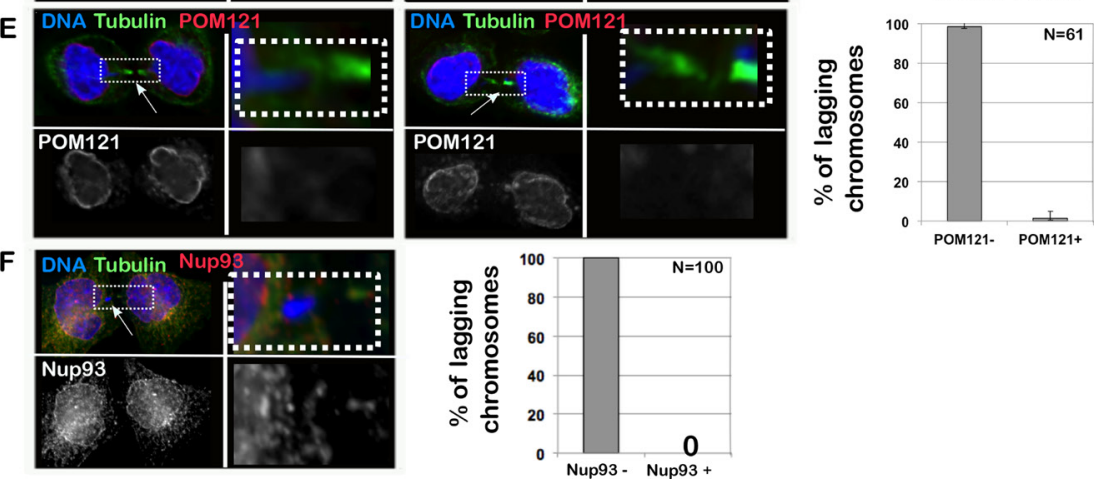

Figure 1: Importin $\beta$ and NPC do not assemble on lagging chromatin in anaphase. HeLa cells were fixed and stained for Lamin $\mathrm{A} / \mathrm{C}$, Importin $\beta$ and several components of the NPC; the presence of staining on chromatin bridges was recorded. (A-F) HeLa cells in telophase/cytokinesis showing chromatin bridges and stained with: Lamin A/C (green) and mAb414 (red) (A); Lamin A/C (green) and Importin $\beta$ (red) (B); INCENP (green) and mAb414 (red) (C); PLK1 (green) and MEL28 (red) (D); GFP:POM121 (red) and $\alpha$-tubulin (green) (E); $\alpha$-tubulin (green) and Nup93 (red) (F); scale bar 10mm. The insets indicate the region of the picture shown at higher magnification in the adjacent column. The graphs represent the distribution of the different classes as mean of 3 independent experiments and the error bars represent SD; the number indicates the total cells counted. 

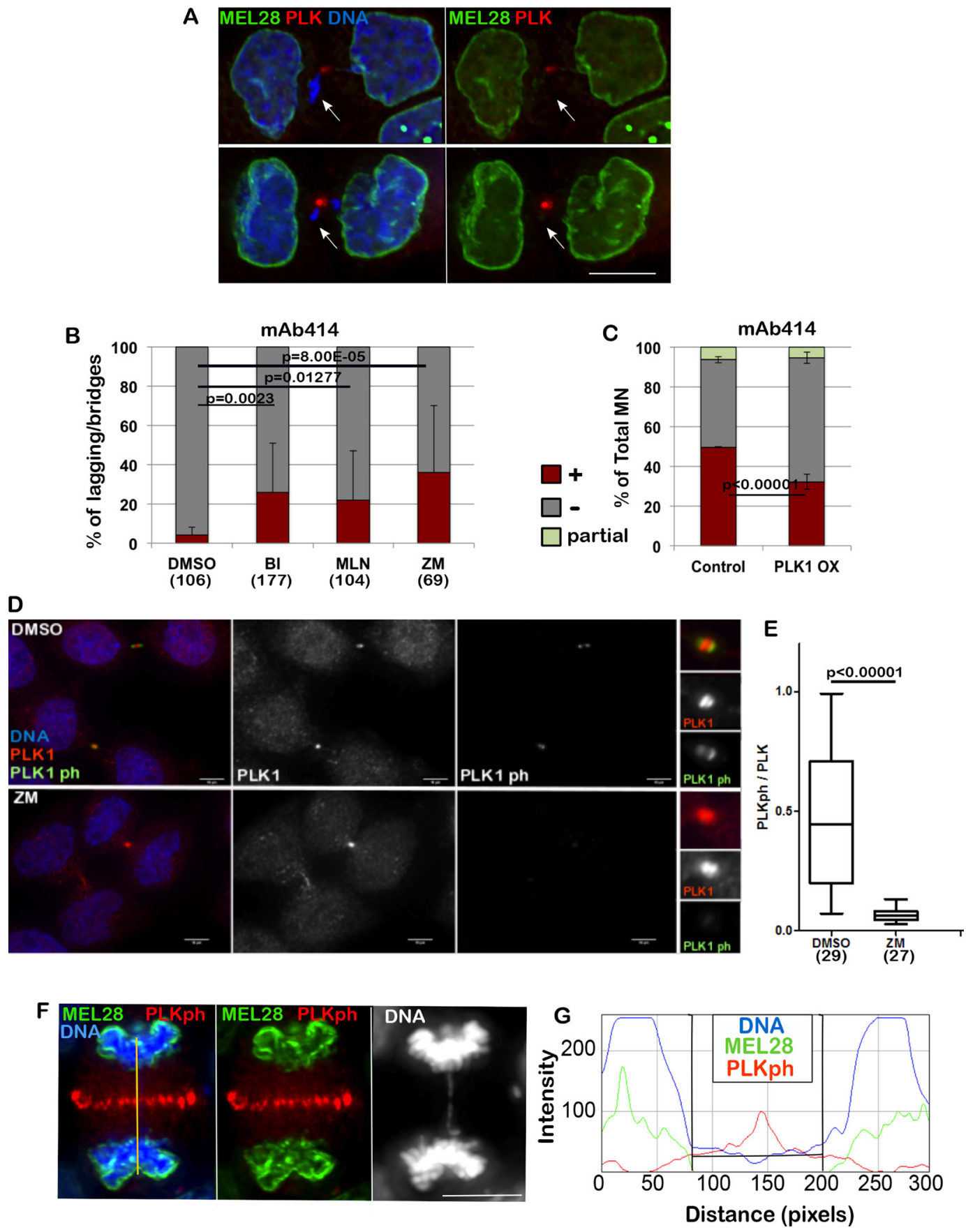

Figure 2: PLK1 and Aurora B regulate the loading of NPC. (A) HeLa cells were fixed and stained for MEL-28 and PLK1. The arrows indicate the lagging chromatin and absence of the NPC component. Scale bar $10 \mu \mathrm{m}$. (B) HeLa cells were treated with DMSO, BI 2536 (BI), MLN0905 (MLN), both targeting PLK1, or ZM447439 (ZM), targeting Aurora B, for $4 \mathrm{~h}$ then fixed and stained for mAb414. The lagging chromatin in telophase cells was analysed for presence or absence of the staining. The error bars indicate the SD between 3 replicates and Fisher exact test was used for statistical analyses. (C) HeLa cells were transiently transfected with mCherry:PLK1 (PLK1 $\mathrm{OX}$ ) and fixed and stained for mAb414 $24 \mathrm{~h}$ post-transfection. The graph shows the percentage of total micronuclei (MN) positive (brown) or negative (grey) for the staining. The error bars indicate the SD between 3 replicates and Fisher exact test was used for the statistical analyses. (D) HeLa cells were treated with either DMSO or ZM447439 (ZM) for 30 min then fixed and stained for PLK1 (red) or PLK1ph (green). Right panel zoom in of the staining at the midbody. Cells at the midbody stage were used for the analyses. Scale bar: $10 \mathrm{~mm}$. (E) quantification of the experiment in (D). Signal intensity ratio between phosphorylated PLK1 and total PLK1 at the midbody in control (DMSO) and Aurora B inhibited (ZM) cells. Mann-Whitney $U$ test was used for statistical analyses. (F) HeLa cells were stained for MEL28 (green) and PLK1ph (red). Anaphase cells showing absence of MEL28 staining on the lagging chromatin. The yellow line indicates the region used for the intensity profile in $(\mathrm{G})$. (G) Intensity profile of the staining in (F) showing accumulation of MELS28 only when PLK1ph decreases. 
a system where spatial clues are removed. Cells blocked in mitosis can be induced to biochemically recapitulate several physiological steps of mitotic exit when cyclindependent kinase 1 (CDK1) is inactivated [9, 29, 30]. To this purpose, we used a chicken DT40 cell line engineered to express an analog sensitive version of CDK1 [31] thus removing the spatial clues from the system. This allele $\left(\mathrm{CDK}{ }^{\mathrm{as}}\right)$ can be specifically inhibited by the bulky ATP analogue 3MB-PP1. The cells were blocked in prometaphase with nocodazole and the accumulation of NPC components around the chromatin was analysed after treatment either with DMSO, BI2536 (to inhibit PLK1), 3MB-PP1 (to inhibit CDK1), ZM447439 (to inhibit Aurora B) or BI2536 + 3M-PP1 (to inhibit both CDK1 and PLK1) for 15 minutes and fixed and stained with mAb414 (Figure 3A-3C) or Importin $\beta$ (Supplementary Figure 2C2D). Similar experiments were conducted also with cells transfected with GFP:Lamin A (DT40 does not express Lamin A) to analyse the behavior of Lamin A and NPC simultaneously. In order to quantify the accumulation of NPC components around the chromatin after inhibition of the kinases, the intensity ratio between the chromosome periphery and cytoplasm staining was calculated at several points around the chromatin (Supplementary Figure 2A, 2B and 2D). A significant enrichment of NPC components at the chromosome periphery was found in treated cells compared to controls (Supplementary Figure 2A, 2B and 2D). In DMSO treated cells, both Lamin A and the NPC or Importin $\beta$ are dispersed in the cytoplasm (Figure 3C-3E). Upon inhibition of CDK1, Lamin A is re-localised onto the chromatin but no further accumulation is produced by co-inhibition of PLK1 (Figure 3D, 3E). Inhibition of CDK1 also triggers NPC recruitment on the chromatin in this system but the additional inhibition of PLK1 produces a nuclear-rim like structure and more substantial accumulation of mAb414 and Importin $\beta$ on the chromosomes (Figure $3 \mathrm{~A}-3 \mathrm{C}$, and Supplementary Figure 2A-2D). A mild effect is observed upon inhibition of PLK1 but not Aurora B alone (Figure $3 \mathrm{~A}-3 \mathrm{C}$, and Supplementary Figure 2B). Inhibition of the kinases was effective within the 15 min treatments as judged by a parallel experiment where cells were treated with single drugs and the proteasome inhibitor MG132 (which blocks cells in metaphase); the mitotic cells were negative for H3S10ph or showed monopolar spindles upon treatment with ZM447439 or BI2536 respectively (Supplementary Figure 3A) as expected when Aurora B or PLK1 are inactive. This data altogether indicates that CDK1 is responsible for maintaining Lamin A off the chromosomes in mitosis but additional phosphorylations by PLK1 or kinases downstream of PLK1 are required to prevent the NPC loading in late mitosis. Taken together the observations so far provided, we can conclude that a conserved mechanism sustained by PLK1 activity (localised at the midzone during mitotic exit) negatively regulates the NPC reformation during anaphase. However this mechanism does not prevent Lamin A recruitment indicating therefore that the two processes are independent and they are monitored by different kinases/phosphatases.

This data reconciles with what is known for Lamin A dynamics in mitosis where CDK1 has been found responsible for phosphorylation of lamins, a prerequisite for NE disassembly. At mitotic entry, the phosphorylation of lamins at specific sites by CDK1 and protein kinase $\mathrm{C}$ (PKC) is required to drive disassembly of the lamina $[2,3,32,33]$ and dephosphorylation of the CDK1 mitotic sites by protein phosphatase 1 (PP1) is required for lamina assembly during the telophase/early G1 transition [34]. It is therefore expected that just the inhibition of CDK1 would license Lamin A deposition on the chromatin.

Given the fact that PLK1 could play a role in NPC re-organisation during mitotic exit, we hypothesized that it could also be important for the dissolution of NPC at mitotic entry. Although a requirement for PLK1 in mitotic entry has been suggested, with $\mathrm{p} 150^{\text {Glued }}$ being one of the substrates involved in this process [23], little is known about PLK1 involvement in the dissolution of the NPC at the onset of mitosis. Nup98, a nucleoporin phosphorylated by multiple kinases, is essential for Nuclear Envelope Breakdown (NEBD) and is also a PLK1 substrate; however, mutation of the two identified PLK1 sites had no impact on NEBD in vitro [4]. Nevertheless, we have noticed that mCherry:PLK1 accumulates at the nuclear periphery in prophase, at a time consistent with its involvement in NE re-organisation (Figure 4A, 4B). A similar observation was reported in Drosophila, where Polo kinase also localises to the NE at mitotic entry [28].

To test if PLK1 was required for the NPC dissolution at mitotic entry, we inhibited CDK1 with $3 \mathrm{MB}-\mathrm{PP} 1$ in the DT40CDK $1^{\text {As }}$ cell line for $6 \mathrm{~h}$ to block cells at the G2/M boundary. After 30 min release, to allow cells enter mitosis, we inhibited PLK1 or Aurora B for $15 \mathrm{~min}$ and analysed the distribution of Importin $\beta$ or mAb414 in mitotic cells (Figure 4C). While in controls and Aurora B inhibited prometaphase/metaphase cells mAb414 and Importin $\beta$ are both diffused and on the spindle, in PLK1 inhibited cells Importin $\beta$ and the NUPs are either still around the condensed chromosomes or in distinct patches within the cytoplasm (Figure 4D-4G). These patches could possibly represent remnants or aggregates of the NE but they do no longer associate with Lamin A. The same results were obtained in HeLa cells. In this system we have inhibited either PLK1 (using either BI2536 or MLN0905) or Aurora $\mathrm{B}$ for $1 \mathrm{~h}$ or $2 \mathrm{~h}$ and then stained the cells for $\mathrm{mAb} 414$ and Lamin A/C. Again the inhibition of only PLK1 causes a delay in the dissolution of the NPC but does not affect the disassembly of Lamin A/C (Figure 4H-4L). These results clearly indicate that also at mitotic entry different kinases are required to complete NEBD and that the breakdown of Lamin A and the NPC follow different pathways. 

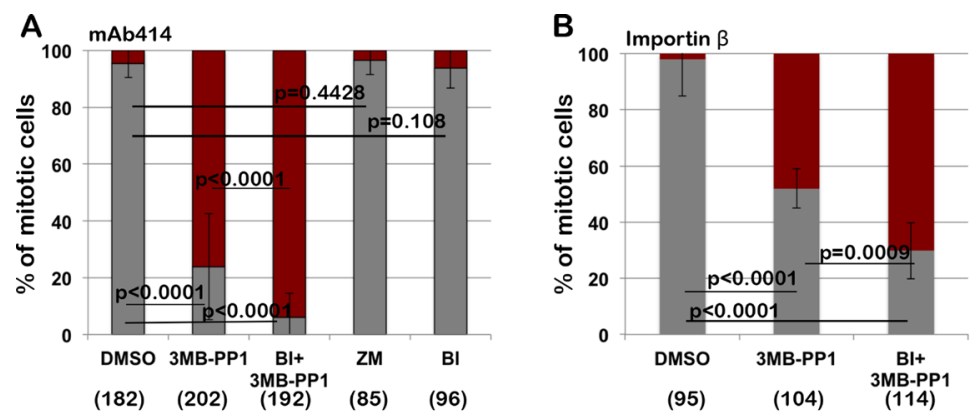

$\square$ Diffused
$\square$ On chromosomes
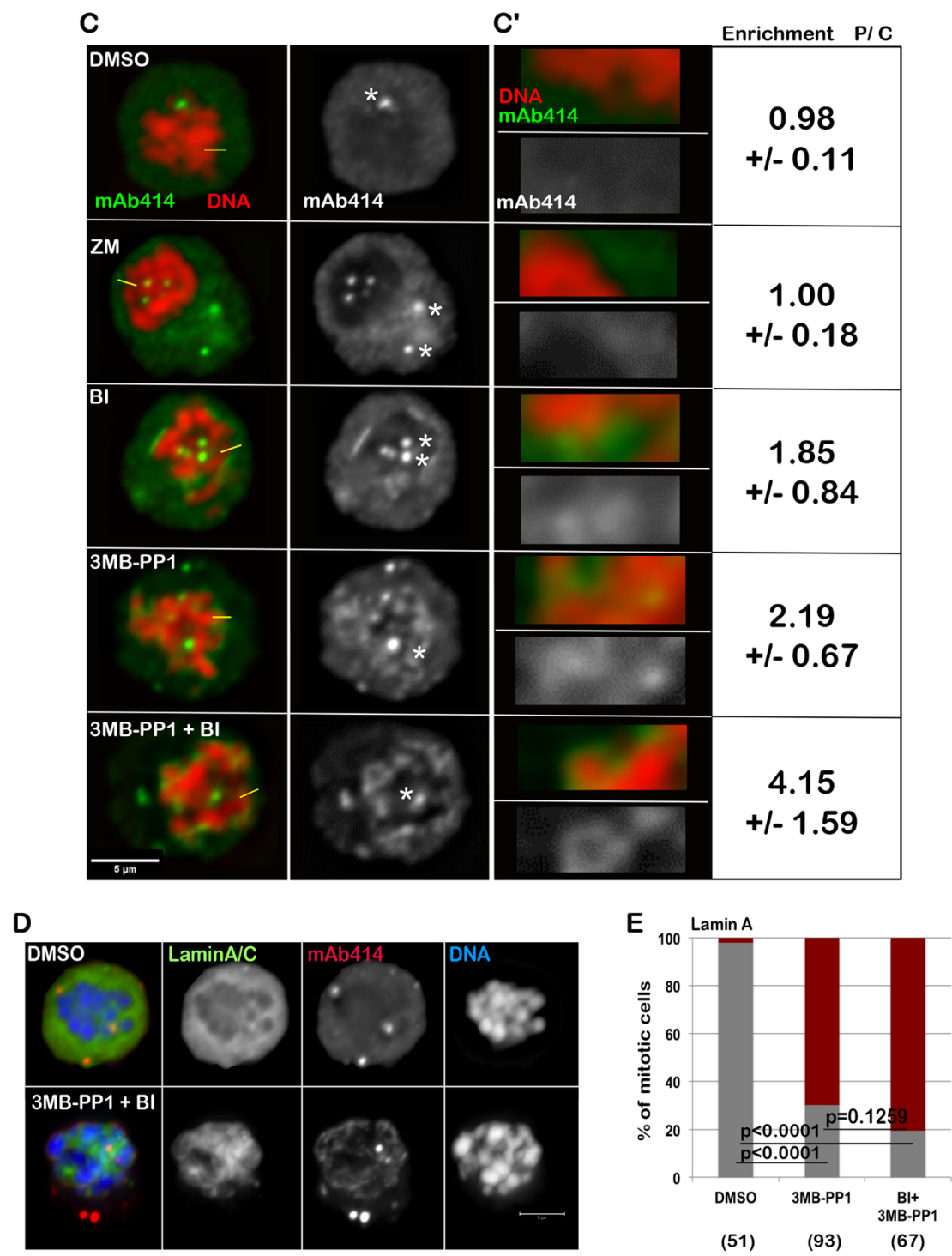

Figure 3: Inhibition of CDK1 and PLK1 in prometaphase cells are both required for the complete loading of the NPC and Importin $\boldsymbol{\beta}$ on the chromatin. (A-B) DT40CDK1 ${ }^{\mathrm{AS}}$ were arrested in nocodazole for $4 \mathrm{~h}$ then treated for $15 \mathrm{~min}$ with DMSO, 3MB-PP1, ZM447439 (ZM), BI2536 (BI) or 3MB-PP1+BI2536 (BI+3MB-PP1). The cells were then stained either for mAb414 (A) or Importin $\beta$ (B) and the localization of the staining was recorded. The data are presented as mean of 3 independent experiments. The error bars indicate the SD between 3 replicates and Fisher exact test was used for the statistical analyses. (C) representative images of the experiment shown in (A). The white asterisks indicate staining at the centrosomes (not considered in the analyses). C': Enlargements of the pictures in (C). Enrichment of signal at the periphery of the chromosomes (P) over Cytoplasm (C) $+/-$ SD is shown. mAb414 (green) and DNA (red). Scale bar $5 \mu \mathrm{m}$. (D) DT40CDK1 ${ }^{\mathrm{AS}}$ were transiently transfected with GFP:LaminA and treated as in A. The localization of GFP:LaminA was recorded. The panels show representative images of cells treated only with DMSO (upper panels) or 3MB-PP1 + BI (bottom panels). (E) quantification of the experiment in (D). The data are presented as mean of 3 independent experiments. The error bars indicate the SD between 3 replicates and Fisher exact test was used for the statistical analyses. 

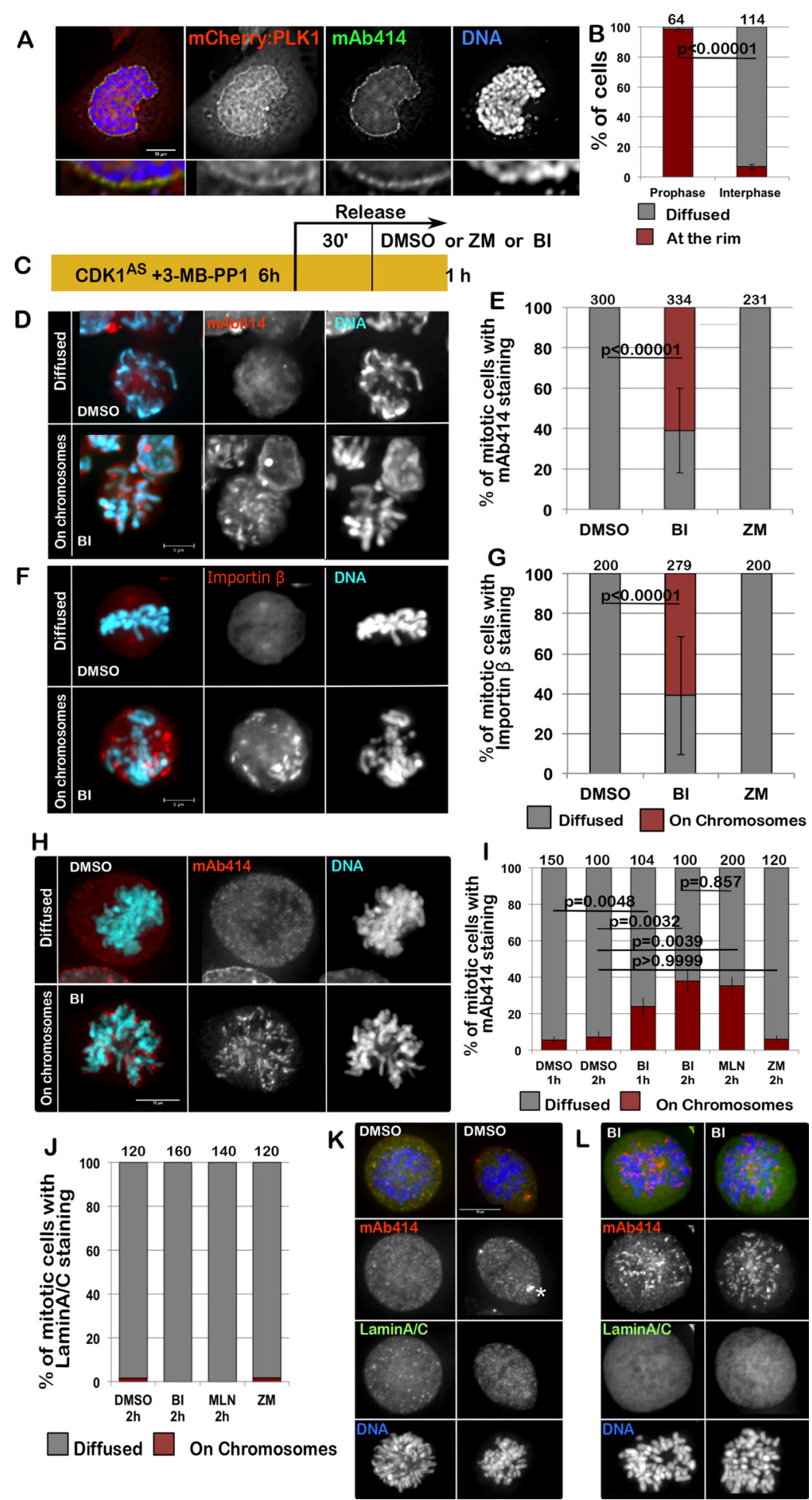

Figure 4: PLK1 is required for the complete disassemble of the NPC at the onset of mitosis. (A) HeLa cells were transfected with mCherry:PLK1 (red) then fixed and stained with mAb414 (green). The nuclear localization of PLK1 was then recorded in interphase and prophase cells. The panels show representative images of a prophase cell. The insets below are magnification of the image above. (B) quantification of the experiment in (A); The data are presented as mean of 3 independent experiments. The error bars indicate the SD between 3 replicates and Fisher exact test was used for the statistical analyses. (C) scheme of the experiment for (D-G). (D-G) DT40CDK ${ }^{\mathrm{AS}}$ after the treatments as in $(\mathrm{C})$ were fixed and stained for $\mathrm{mAb} 414$ or Importin $\beta$. Inhibition of PLK1 compromises the dispersal of the NPC in mitotic cells. The panels show representative images of mAb414 staining (D) or Importin $\beta$ (F) in DMSO (diffused) or BI (on chromosomes)-treated cells (D); (E-G) quantification of the experiments in D and F. The data are presented as mean of 3 independent experiments. The error bars indicate the SD between 3 replicates and Fisher exact test was used for the statistical analyses. (H-I) HeLa cells were treated with either DMSO, BI 2536 (BI), MLN0905 (MLN) or ZM447439 (ZM) for 1 or $2 \mathrm{~h}$ then the cells were fixed and stained for 
mAb414 (red). The distribution of mAb414 classified as "diffused" or "on chromosomes" was quantified (I). Representative images of a "diffuse" (upper panels) or "on chromosome" (lower panels) pattern are presented in (H). (J-L) HeLa cells were treated with either DMSO, BI 2536 (BI), MLN0905 (MLN) or ZM447439 (ZM) for 1 or $2 \mathrm{~h}$ then the cells were fixed and stained for mAb414 (red) and Lamin A/C (green). The distribution of Lamin A classified as "diffused" or "on chromosomes" was quantified (J). The data are presented as mean of 3 independent experiments. The error bars indicate the SD between 3 replicates and Fisher exact test was used for the statistical analyses. Representative images of DMSO (K) or BI (L) -treated cells are shown. The white asterisk in (K) indicates staining at the centrosomes.

\section{DISCUSSION}

Here we have shown that Lamina A/C and NPC dissolution and reformation are controlled by different mechanisms. CDK1 is necessary for Lamin A breakdown and its inhibition is sufficient for targeting it onto the chromosomes. However, the NPCs require the additional phosphorylation by PLK1 for their full dispersal at mitosis onset, and PLK1 inactivation for the re-assembly at mitotic exit (Figure 5). These observations are consistent with the fact that mitotic phosphorylation of several nucleoporins depends on PLK1 as suggested by published proteomic studies [35-37] and supported by previous observations linking lack of PLK1 to delays of NEBD in human cells [18], C. elegans $[38,39]$ and in mouse oocytes maturation [40]. However this is the first evidence that NPC and Lamin $\mathrm{A} / \mathrm{C}$ are behaving in an independent manner. At mitotic exit the NPC re-assembly is spatially controlled by the presence of active PLK1 whereas Lamin A is regulated via a "clock" mechanism based on CDK1 inactivation without any topological control; in fact it is not important where the chromatin is within the anaphase cell to be competent for Lamin A binding. Although the PLK1-nonphosphorylable mutants of Nup98 did not show an effect in inhibiting NEBD in vitro [4], it is possible that several phosphorylation events on different nucleoporins have additive effects and altogether contribute to the full NPC disassembly. Therefore, mutations in single components might not be sufficient to produce major defects.

This study therefore provides mechanistic insights into the pathway on NE re-organisation in vertebrates. In the future, identifying the specific counteracting phosphatases will be an important aspect to pursue in order to understand the full network regulating NEBD and NER. It is possible that PLK1 negatively regulates the activity of phosphatases as well. Hints towards this direction are that some NPC components have been shown to be substrates for PP2A-B55 [41] but the sites identified are not the ones sensitive to PLK1 inhibition [37].

It is worth noting that Repo-Man/PP1, which activity is regulated by Aurora B [42], can bind to Nup153 [43] and is important for Importin $\beta$ recruitment on the lagging chromatin [9]; this complex could also represent a potential phosphatase candidate.

Importantly, our findings have also major implications in genome stability. As we have shown, the $\mathrm{MN}$ that originate from merotelic attached chromosomes are inevitably going to have a compromised nuclear barrier from the start as their import/export machinery is impaired. These MN will then be a major source of genomic instability as elegantly demonstrated recently by the Pellman Lab [14]. These findings could therefore add extra value to the use of PLK1 inhibitors for the treatment of specific cancer types arising from $\mathrm{MN}$ formation.

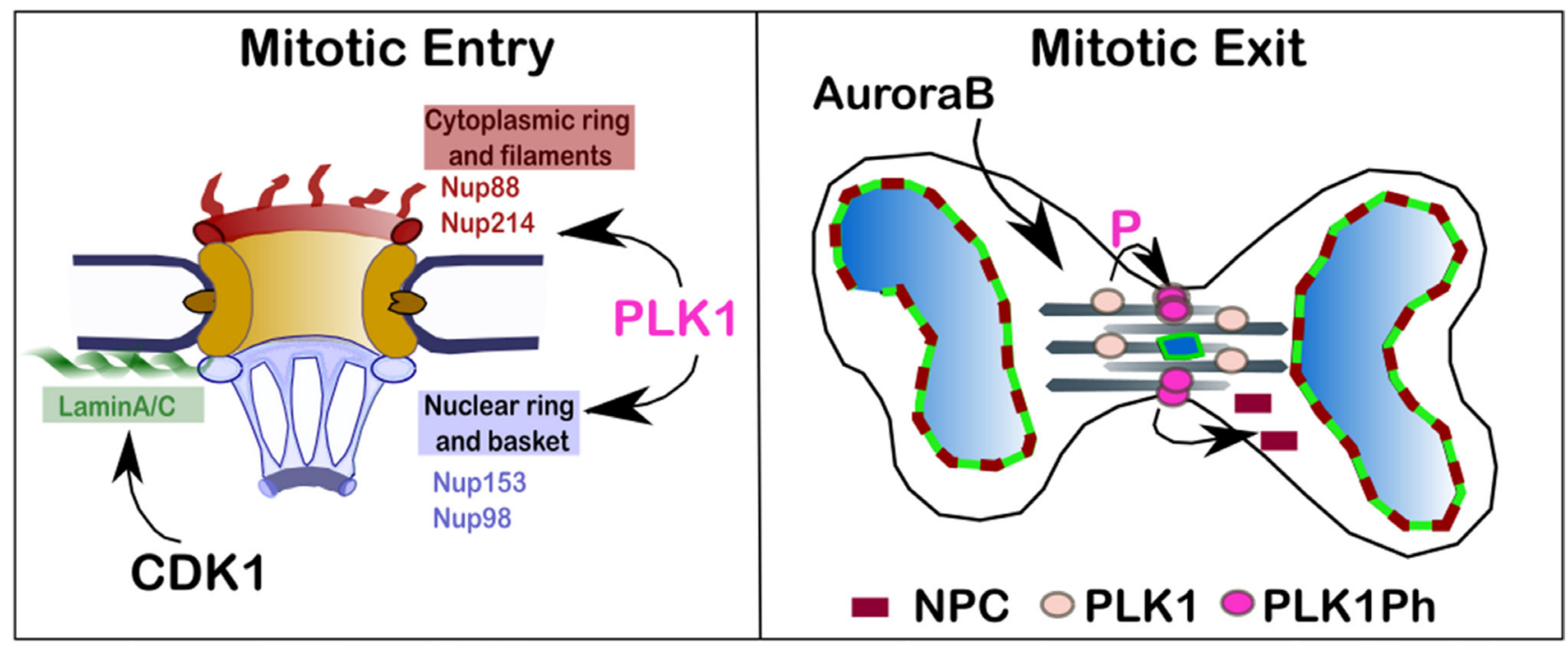

Figure 5: Proposed model for the regulation of NPC re-organisation in mitosis. At mitotic entry PLK1 localises at the nuclear rim in prophase where phosphorylates a subset of nucleoporins contributing to the disassembly of the NPC. CDK1 phosphorylates Lamin A and is important for the NE disassembly. At mitotic exit, CDK1 inhibition is essential for triggering NE re-assembly and is sufficient for targeting Lamin A/C to the chromatin in vertebrates. The complete NPC re-assembly requires, in addition, the inhibition of PLK1 which activity is sustained by Aurora B at the cleavage furrow. 


\section{MATERIALS AND METHODS}

\section{Cell Culture}

HeLa cells were cultured in DMEM supplemented with $10 \%$ FBS and DT40 cells were cultured in RPMI1640 supplemented with $10 \%$ FBS and $1 \%$ chicken serum at $37^{\circ} \mathrm{C}$ and $39^{\circ} \mathrm{C}$ respectively in $5 \% \mathrm{CO}_{2}$. DT40 cells DT40CDK1 ${ }^{\mathrm{AS}}$ cells were provided by Dr Helfrid Hochegger (Sussex, UK). HeLa GFP:POM121 cell line was kindly provided by Kathrine Ullamn (Utha, USA).

Transient transfections for DT40 were conducted as previously described [9]. For synchronization, DT40 cells DT40CDK1 ${ }^{\text {AS }}$ cells were treated with $100 \mathrm{mM}$ of 3MB-PP1 (Millipore) for $4 \mathrm{~h}$ or with $100 \mathrm{ng} / \mathrm{ml}$ nocodazole (Sigma).

BI2536 (Stratech), ZM447439 and Y-27632 (Calbiochem), MLN0905 (Sellckechem) were used at $100 \mathrm{nM}, 2 \mu \mathrm{M}, 40 \mu \mathrm{M}$, and $35 \mu \mathrm{M}$ respectively for the time indicated in each experiment.

mCherry-PLK1-N-16 was a gift from Michael Davidson (Addgene plasmid 55119)

\section{Immunofluorescence and microscopy}

For immunofluorescence, cells were fixed in $4 \%$ PFA and processed as previously described [9]; antibody incubations were carried out in 1\% BSA-phosphatebuffered saline (PBS) with 1\%BSA and $0.01 \%$ TWEEN for $1 \mathrm{~h}$ at $37^{\circ} \mathrm{C}$.

Fluorescence-labelled secondary antibodies were applied at 1:200 (Jackson ImmunoResearch).

The primary antibodies were used as follows: Anti Nup153 (mouse QE5, Abcam, ab24700) 1:300; mAb414 (mouse Covance 1:500, MMS-120R); AntiImportin $\beta$ (monoclonal 3E9, Abcam, ab2811) 1:1000; anti-Lamin A/C (rabbit monoclonal, Abcam ab108595); anti-Ser10ph (rabbit, Upstate Biotechnoloy,\#06-570) 1:200; anti-atubulin (monoclonal B512, Sigma, T5168) 1:1000; anti-PLK1 (Abcam, ab175808) 1:1000; antiPLK1ph (Abcam, ab39068) 1:300; anti-INCENP (gift from WC Earnshaw, Edinburgh) 1:500; Mel28 (Gift from M. Platani, Edinburgh) 1:500; Nup93 (Santa Cruz, GC292099) 1:100.

3D data sets were acquired on a wide-field microscope (NIKON Ti-E super research Live Cell imaging system) with a NA 1.45 Plan Apochromat lens. The data sets were deconvolved with NIS-Element AR software. 3D data sets were converted to Quick Projections or presented as single plane, exported as TIFF files, and imported into Adobe Photoshop for final presentation.

For quantification of the staining (Figure 2C-2D), an area containing the midbody was select and the total staining in the red and blue channel was measured. Another set of measurements was obtained from the same areas in a region outside the cell (background) and subtracted by the intensities in the midbody.
For the quantification of the signal at the chromosome periphery versus cytoplasm (Figure 3 and C, C' and Supplementary Figure 2A-2D), a line scan was generated in several points around the chromosomes (as in Supplementary Figure 2A). The ratio between intensity of the signal recorded at the chromosome periphery and the average cytoplasm intensity was calculated per position at each nucleus. The values obtained were compared (Mann-Whitney $U$ test) with the DMSO treated cells and, if significantly different, recorded as "on chromosomes".

The graphs were generated in Excel or R-studio, exported as png and imported in Inkscape for figure presentation.

\section{Data Availability}

The microscopy data are available from the corresponding author upon request and will be released via figshare.

\section{Author contributions}

IC and RSG were responsible for conducting experiments, data analyses and contributed to manuscript writing; LL and LDG were responsible for conducting experiments; PV supervised the project and was responsible for manuscript conceptualization, data interpretation and manuscript writing.

\section{ACKNOWLEDGMENTS}

We would like to thank Mar Carmena (Edinburgh), Eric Shirmer (Edinburgh) and Joanna M Bridger (Brunel University London) for critical reading of the manuscript.

\section{CONFLICTS OF INTEREST}

The authors declare no conflicts of interest.

\section{FUNDING}

The Vagnarelli Lab is funded by BBSRC (grant BB/ K017632/1 to PV). MLDG was supported by a Placement ERASMUS fellowship. RSG is supported by an Isambard kingdom scholarship.

\section{REFERENCES}

1. de Castro IJ, Gokhan E, Vagnarelli P. Resetting a functional G1 nucleus after mitosis. Chromosoma. 2016. https://doi. org/10.1007/s00412-015-0561-6.

2. Peter M, Nakagawa J, Doree M, Labbe JC, Nigg EA. In vitro disassembly of the nuclear lamina and $\mathrm{M}$ phasespecific phosphorylation of lamins by cde2 kinase. Cell. 1990; 61:591-602. 
3. Heald R, McKeon F. Mutations of phosphorylation sites in lamin A that prevent nuclear lamina disassembly in mitosis. Cell. 1990; 61:579-89.

4. Laurell E, Beck K, Krupina K, Theerthagiri G, Bodenmiller B, Horvath P, Aebersold R, Antonin W, Kutay U. Phosphorylation of Nup98 by multiple kinases is crucial for NPC disassembly during mitotic entry. Cell. 2011; 144:539-50. https://doi.org/10.1016/j.cell.2011.01.012.

5. Dultz E, Ellenberg J. Live imaging of single nuclear pores reveals unique assembly kinetics and mechanism in interphase. J Cell Biol. 2010; 191:15-22. https://doi. org/10.1083/jcb.201007076.

6. Weberruss M, Antonin W. Perforating the nuclear boundary - how nuclear pore complexes assemble. J Cell Sci. 2016; 129:4439-47. https://doi.org/10.1242/jcs.194753.

7. LaJoie D, Ullman KS. Coordinated events of nuclear assembly. Curr Opin Cell Biol. 2017; 46:39-45. https://doi. org/10.1016/j.ceb.2016.12.008.

8. Steen RL, Beullens M, Landsverk HB, Bollen M, Collas P. AKAP149 is a novel PP1 specifier required to maintain nuclear envelope integrity in G1 phase. J Cell Sci. 2003; 116:2237-46. https://doi.org/10.1242/jcs.00432.

9. Vagnarelli P, Ribeiro S, Sennels L, Sanchez-Pulido L, de Lima Alves F, Verheyen T, Kelly DA, Ponting CP, Rappsilber J, Earnshaw WC. Repo-Man coordinates chromosomal reorganization with nuclear envelope reassembly during mitotic exit. Dev Cell. 2011; 21:328-42. https://doi.org/10.1016/j.devcel.2011.06.020.

10. Asencio C, Davidson IF, Santarella-Mellwig R, Ly-Hartig TB, Mall M, Wallenfang MR, Mattaj IW, Gorjanacz M. Coordination of kinase and phosphatase activities by Lem4 enables nuclear envelope reassembly during mitosis. Cell. 2012; 150:122-35. https://doi.org/10.1016/j. cell.2012.04.043.

11. Afonso O, Matos I, Pereira AJ, Aguiar P, Lampson MA, Maiato H. Feedback control of chromosome separation by a midzone Aurora B gradient. Science. 2014; 345:332-6. https://doi.org/10.1126/science.1251121.

12. Karg T, Warecki B, Sullivan W. Aurora B-mediated localized delays in nuclear envelope formation facilitate inclusion of late-segregating chromosome fragments. Mol Biol Cell. 2015; 26:2227-41. https://doi.org/10.1091/mbc.E15-01-0026.

13. Hatch EM, Fischer AH, Deerinck TJ, Hetzer MW. Catastrophic nuclear envelope collapse in cancer cell micronuclei. Cell. 2013; 154:47-60. https://doi. org/10.1016/j.cell.2013.06.007.

14. Zhang CZ, Spektor A, Cornils H, Francis JM, Jackson EK, Liu S, Meyerson M, Pellman D. Chromothripsis from DNA damage in micronuclei. Nature. 2015; 522:179-84. https:// doi.org/10.1038/nature14493.

15. Utani K, Kohno Y, Okamoto A, Shimizu N. Emergence of micronuclei and their effects on the fate of cells under replication stress. PLoS One. 2010; 5:e10089. https://doi. org/10.1371/journal.pone.0010089.
16. Cimini D, Howell B, Maddox P, Khodjakov A, Degrassi F, Salmon ED. Merotelic kinetochore orientation is a major mechanism of aneuploidy in mitotic mammalian tissue cells. J Cell Biol. 2001; 153:517-27.

17. Schwartz TU. The Structure Inventory of the Nuclear Pore Complex. J Mol Biol. 2016; 428:1986-2000. https://doi. org/10.1016/j.jmb.2016.03.015.

18. Lenart P, Petronczki M, Steegmaier M, Di Fiore B, Lipp JJ, Hoffmann M, Rettig WJ, Kraut N, Peters JM. The small-molecule inhibitor BI 2536 reveals novel insights into mitotic roles of polo-like kinase 1. Curr Biol. 2007; 17:304-15. https://doi.org/10.1016/j.cub.2006.12.046.

19. Steegmaier M, Hoffmann M, Baum A, Lenart P, Petronczki M, Krssak M, Gurtler U, Garin-Chesa P, Lieb S, Quant J, Grauert M, Adolf GR, Kraut N, et al. BI 2536, a potent and selective inhibitor of polo-like kinase 1, inhibits tumor growth in vivo. Curr Biol. 2007; 17:316-22. https://doi. org/10.1016/j.cub.2006.12.037.

20. Moshe Y, Bar-On O, Ganoth D, Hershko A. Regulation of the action of early mitotic inhibitor 1 on the anaphasepromoting complex/cyclosome by cyclin-dependent kinases. J Biol Chem. 2011; 286:16647-57. https://doi. org/10.1074/jbc.M111.223339.

21. Liang H, Esposito A, De S, Ber S, Collin P, Surana U, Venkitaraman AR. Homeostatic control of polo-like kinase-1 engenders non-genetic heterogeneity in G2 checkpoint fidelity and timing. Nat Commun. 2014; 5:4048. https://doi.org/10.1038/ncomms5048.

22. Lera RF, Burkard ME. High mitotic activity of Pololike kinase 1 is required for chromosome segregation and genomic integrity in human epithelial cells. J Biol Chem. 2012; 287:42812-25. https://doi.org/10.1074/jbc. M112.412544.

23. Li H, Liu XS, Yang X, Song B, Wang Y, Liu X. Polo-like kinase 1 phosphorylation of p150Glued facilitates nuclear envelope breakdown during prophase. Proc Natl Acad Sci U S A. 2010; 107:14633-8. https://doi.org/10.1073/ pnas. 1006615107.

24. Burkard ME, Santamaria A, Jallepalli PV. Enabling and disabling polo-like kinase 1 inhibition through chemical genetics. ACS Chem Biol. 2012; 7:978-81. https://doi. org/10.1021/cb200551p.

25. Ditchfield C, Johnson VL, Tighe A, Ellston R, Haworth C, Johnson T, Mortlock A, Keen N, Taylor SS. Aurora B couples chromosome alignment with anaphase by targeting BubR1, Mad2, and Cenp-E to kinetochores. J Cell Biol. 2003; 161:267-80. https://doi.org/10.1083/jcb.200208091.

26. de Groot CO, Hsia JE, Anzola JV, Motamedi A, Yoon M, Wong YL, Jenkins D, Lee HJ, Martinez MB, Davis RL, Gahman TC, Desai A, Shiau AK. A Cell Biologist's Field Guide to Aurora Kinase Inhibitors. Front Oncol. 2015; 5:285. https://doi.org/10.3389/fonc.2015.00285.

27. Royou A, Gagou ME, Karess R, Sullivan W. BubR1- and Polo-coated DNA tethers facilitate poleward segregation of 
acentric chromatids. Cell. 2010; 140:235-45. https://doi. org/10.1016/j.cell.2009.12.043.

28. Carmena M, Pinson X, Platani M, Salloum Z, Xu Z, Clark A, Macisaac F, Ogawa H, Eggert U, Glover DM, Archambault V, Earnshaw WC. The chromosomal passenger complex activates Polo kinase at centromeres. PLoS Biol. 2012; 10:e1001250. https://doi.org/10.1371/ journal.pbio.1001250.

29. Kachaner D, Pinson X, El Kadhi KB, Normandin K, Talje L, Lavoie H, Lepine G, Carreno S, Kwok BH, Hickson GR, Archambault V. Interdomain allosteric regulation of Polo kinase by Aurora B and Map205 is required for cytokinesis. J Cell Biol. 2014; 207:201-11. https://doi.org/10.1083/ jcb.201408081.

30. Ma S, Vigneron S, Robert P, Strub JM, Cianferani S, Castro A, Lorca T. Greatwall dephosphorylation and inactivation upon mitotic exit is triggered by PP1. J Cell Sci. 2016; 129:1329-39. https://doi.org/10.1242/jcs.178855.

31. Hochegger H, Dejsuphong D, Sonoda E, Saberi A, Rajendra E, Kirk J, Hunt T, Takeda S. An essential role for Cdk1 in $\mathrm{S}$ phase control is revealed via chemical genetics in vertebrate cells. J Cell Biol. 2007; 178:257-68. https://doi. org/10.1083/jcb.200702034.

32. Collas P, Thompson L, Fields AP, Poccia DL, Courvalin JC. Protein kinase C-mediated interphase lamin B phosphorylation and solubilization. J Biol Chem. 1997; 272:21274-80.

33. Gerace L, Blobel G. The nuclear envelope lamina is reversibly depolymerized during mitosis. Cell. 1980; 19:277-87.

34. Thompson LJ, Bollen M, Fields AP. Identification of protein phosphatase 1 as a mitotic lamin phosphatase. J Biol Chem. 1997; 272:29693-7.

35. Sauer G, Korner R, Hanisch A, Ries A, Nigg EA, Sillje HH. Proteome analysis of the human mitotic spindle. Mol Cell Proteomics. 2005; 4:35-43. https://doi.org/10.1074/mcp. M400158-MCP200.

36. Sanchez-Quiles V, Mora MI, Segura V, Greco A, Epstein AL, Foschini MG, Dayon L, Sanchez JC, Prieto J, Corrales FJ, Santamaria E. HSV-1 Cgal+ infection promotes quaking
RNA binding protein production and induces nuclearcytoplasmic shuttling of quaking I-5 isoform in human hepatoma cells. Mol Cell Proteomics. 2011; 10:M111 009126. https://doi.org/10.1074/mcp.M111.009126.

37. Kettenbach AN, Schweppe DK, Faherty BK, Pechenick D, Pletnev AA, Gerber SA. Quantitative phosphoproteomics identifies substrates and functional modules of Aurora and Polo-like kinase activities in mitotic cells. Sci Signal. 2011; 4:rs5. https://doi.org/10.1126/scisignal.2001497.

38. Chase D, Serafinas C, Ashcroft N, Kosinski M, Longo D, Ferris DK, Golden A. The polo-like kinase PLK1 is required for nuclear envelope breakdown and the completion of meiosis in Caenorhabditis elegans. Genesis. 2000; 26:26-41.

39. Rahman MM, Munzig M, Kaneshiro K, Lee B, Strome S, Muller-Reichert T, Cohen-Fix O. Caenorhabditis elegans polo-like kinase PLK-1 is required for merging parental genomes into a single nucleus. Mol Biol Cell. 2015; 26:4718-35. https://doi.org/10.1091/mbc.E15-04-0244.

40. Solc P, Kitajima TS, Yoshida S, Brzakova A, Kaido M, Baran V, Mayer A, Samalova P, Motlik J, Ellenberg J. Multiple requirements of PLK1 during mouse oocyte maturation. PLoS One. 2015; 10:e0116783. https://doi. org/10.1371/journal.pone.0116783.

41. Cundell MJ, Hutter LH, Nunes Bastos R, Poser E, Holder J, Mohammed S, Novak B, Barr FA. A PP2A-B55 recognition signal controls substrate dephosphorylation kinetics during mitotic exit. J Cell Biol. 2016; 214:539-54. https://doi. org/10.1083/jcb.201606033.

42. Kumar GS, Gokhan E, De Munter S, Bollen M, Vagnarelli P, Peti W, Page R. The Ki-67 and RepoMan mitotic phosphatases assemble via an identical, yet novel mechanism. Elife. 2016; 5. https://doi.org/10.7554/ eLife.16539.

43. de Castro IJ, Budzak J, Di Giacinto ML, Ligammari L, Gokhan E, Spanos C, Moralli D, Richardson C, de Las Heras JI, Salatino S, Schirmer EC, Ullman KS, Bickmore WA, et al. Repo-Man/PP1 regulates heterochromatin formation in interphase. Nat Commun. 2017; 8:14048. https://doi.org/10.1038/ncomms14048. 